\title{
DYNAMIC THERMAL BUILDING ANALYSIS WITH CFD - MODELLING RADIATION
}

\author{
Zitzmann T. ${ }^{1}$, Pfrommer P. ${ }^{1}$, Cook M.J. ${ }^{2}$ \\ ${ }^{1}$ University of Applies Sciences Coburg, Germany \\ ${ }^{2}$ Institute of Energy and Sustainable Development, De Montfort University, UK
}

\begin{abstract}
$\underline{\text { ABSTRACT }}$
In an attempt to reduce the high computational efforts for dynamic thermal simulations using Computational Fluid Dynamics (CFD) the authors have recently developed an adaptive freeze-flow method (i.e. freezing of flow equations over variable time periods). This paper documents the work that has been carried out to predict the radiative surface heat transfer in dynamic thermal building processes using CFD.

The Monte Carlo and Discrete Transfer radiation models were investigated and results compared with analytical solutions. The Discrete Transfer model has shown good performance whereas an unrealistic radiation distribution on the surfaces was observed for the Monte Carlo model. A further investigation of the Discrete Transfer model for the cooling of a solid wall has shown that the adaptive freeze-flow method is an efficient and accurate way to carry out dynamic thermal CFD simulations which involve radiation.
\end{abstract}

\section{KEYWORDS}

Radiation modelling; dynamic CFD; conjugate heat transfer; freeze-flow method.

\section{INTRODUCTION}

Zonal programs are commonly used for predicting the dynamic thermal performance of buildings. However, due to their assumption of perfectly mixed thermal conditions and one-dimensional heat conduction in the building fabric, this can lead to significant prediction errors. Although CFD programs potentially lead to greater accuracy as they are based on the Finite Volume Method (Versteeg and Malalasekera 1995), they are often restricted to steady-state problems or to only few minutes of simulation due to the high requirement of computational resources (e.g. see Groleau 1997, Haupt 2001 and Farvarolo et al 2005).

In an attempt to overcome this limitation, an invariable freeze-flow method was developed by Somarathne et al (2002) The 'freezing' of the flow equations for fixed time periods enabled dynamic CFD simulations to be completed in about $80 \%$ of the time required for a full dynamic CFD simulation in which all equations were calculated continuously at every time step. Prediction errors were small. An enhanced, adaptive freeze-flow method was developed by Zitzmann et al (2007a) which 'freezes' the flow equations for variable time periods. This method further reduced the computational effort compared with a fully dynamic CFD simulation. However, the method was verified for conjugate heat transfer processes in which radiative heat transfer was not considered. Radiative surface heat transfer often dominates the convective surface heat transfer in buildings (e.g. Ghatti and Autif 2002 and Sharma et al 2007). It is thus still unknown how the method behaves if radiation is considered.

CFD is mainly used to predict the air flow patterns using steady-state schemes (e.g. Cook et al 2003 and Farvarolo et al 2005). Since the thermal mass of the enclosure is often not modelled and fixed thermal boundary conditions are assigned to the surfaces to reduce the compuational effort, radiation modelling is often neglected. Thus, little information has been published which provides reccomendations on the use of radiation models and appropriate parameter settings for dynamic building simulations using CFD. This paper documents the work that has been carried out to investigate the influence of radiation models in CFD for the application to dynamic thermal simulation. Predictions were verified by comparing the results with analytical solutions for two simple test models. Radiative heat transfer was then implemented in a room model of conjugate heat transfer for dynamic CFD simulations to verify the adaptive freeze-flow method. Simulation results have been compared with a fully transient CFD simulation.

In this work the CFD program ANSYS CFX (ANSYS 2005) has been used.

\section{$\underline{\text { ANALYTICAL METHODS }}$}

The radiative heat flux, E, emitted from a grey body is derived from the law of Stefan-Boltzmann and is calculated as follows:

$$
E=\varepsilon \cdot \sigma \cdot T^{4}
$$

where $\varepsilon$ is the radiative emissivity, $\sigma$ is the StefanBoltzmann constant and $\mathrm{T}$ the surface temperature of the body.

The radiative heat exchange between surfaces can be analytically derived for certain simple cases (see Wagner 1998). Two analytical cases which have 
been used for comparison with numerical solutions in this work are described as follows. The analytical solutions are based on the assumption that the surfaces are grey and diffuse reflective.

\section{Parallel plates (Infinite surfaces)}

For two parallel infinite large surfaces, with rays reflected multiple times between the surfaces before the reflected radiation intensity approaches zero, e.g. for $\varepsilon<<1$, Eq. (2) is used for calculating the radiative heat exchange (Wagner 1998):

$$
\dot{q}_{i k}=\frac{\sigma}{\frac{1}{\varepsilon_{i}}+\frac{1}{\varepsilon_{i}}} \cdot\left(T_{i}^{4}-T_{k}^{4}\right)
$$

\section{Enclosure (Finite surfaces)}

For the case of finite surfaces in a realistic room, the total incident radiation, $B_{i}$, at a surface is the sum of the emitted and reflected radiation from $n$ participating surfaces (Sharma et al 2007):

$$
B_{i}=\sum_{n}\left(\varphi_{k i} \cdot \sigma \cdot \varepsilon_{k} \cdot T_{k}^{4}+\varphi_{k i} \cdot\left(1-\varepsilon_{k}\right) \cdot B_{k}\right)
$$

where $\varphi_{\mathrm{ik}}$ is the view factor between two surfaceelements which may be divided into sub-elements to increase accuracy. For simple geometries, typical view factors are known, which accurately consider the spatial distribution of the geometrical conditions (see for example Wagner 1998). Eq. (3) leads to an equation-system which couples all surfaces. An implicit solution of the system in a matrix becomes difficult due to the non-linearity of the temperature terms. If the surface temperatures are known, the system becomes linear and can be solved with standard matrix solution techniques giving the total incident radiation, $B_{i}$, at each surface. Due to the law of Kirchhoff $(\varepsilon=\alpha)$ the radiative heat absorbed at each surface is given by the difference between the incident and emitted radiation using Eq. (4):

$\dot{q}_{i k}=\varepsilon_{i} \cdot B_{i}-\varepsilon_{i} \cdot \sigma \cdot T_{i}^{4}$

No heat can be stored at adiabatic wall conditions and Eq. (4) becomes zero. The equation simplifies to Eq. (5):

$\sigma \cdot T_{i}^{4}=B_{i}$

Inserting Eq. (5) into Eq. (3) the emissivities and temperatures disappear in the equations for the adiabatic surfaces. For the calculation of radiative heat transfer only the temperatures of the other, nonadiabatic surfaces need to be known which simplifies the solution of the matrix. The resulting surface temperatures for the adiabatic walls are then determined by inserting the relevant $B_{i}$ in Eq. (5).

\section{RADIATION MODELS IN CFX}

In CFX, radiation is represented by particles which are tracked through the air domain using a raytracing method. The spectral radiative transport equation used in CFX (ANSYS 2005) is:

$$
\begin{aligned}
& \frac{d I_{v}(\vec{r}, \vec{s})}{d s}=-\left(K_{a v}+K_{s v}\right) \cdot I_{v}(\vec{r}, \vec{s})+K_{a v} \cdot I_{b}(v, T)+ \\
& +\frac{K_{s v}}{4 \pi} \int_{4 \pi} d I v\left(\vec{r}, \vec{s}^{\prime}\right) \cdot \Phi \cdot\left(\vec{s} \bullet \vec{s}^{\prime}\right) \cdot d \Omega^{\prime}+S \\
& \mathrm{I}_{\mathrm{b}} \quad \text { blackbody emission intensity }\left[\mathrm{W} / \mathrm{m}^{2}\right] \\
& \mathrm{I}_{\mathrm{V}} \quad \text { spectral emission intensity }\left[\mathrm{W} / \mathrm{m}^{2}\right] \\
& \vec{r} \quad \text { position vector [m] } \\
& \vec{S} \quad \text { direction vector }[\mathrm{m}] \\
& \mathrm{s} \quad \text { path length [m] } \\
& \mathrm{K}_{\mathrm{a}} \quad \text { absorption coefficient [-] } \\
& \mathrm{K}_{\mathrm{s}} \quad \text { scattering coefficient [-] } \\
& \mathrm{V} \text { frequency }\left[\mathrm{s}^{-1}\right] \\
& \mathrm{T} \quad \text { local absolute temperature [T] } \\
& \Phi \quad \text { in-scattering phase function } \\
& \Omega \quad \text { solid angle [rad] } \\
& \mathrm{S} \quad \text { a source term }\left[\mathrm{W} / \mathrm{m}^{2}\right]
\end{aligned}
$$

The formal solution of the radiative transfer equation is very time consuming and achieved in CFX by using approximate models for the directional and spectral dependencies.

The spectral approximation used in this work is that the medium which takes part at radiation heat transfer is non-scattering and grey (i.e. independent of the wavelength). Opening boundary conditions are considered as fully transparent to radiation. Walls are treated as diffuse emitting and reflecting opaque boundaries whereas symmetry planes are treated as diffusely emitting and specular reflecting boundaries in CFX.

Two directional radiation models are recommended for optically thin media (i.e. transparent to radiation at wavelengths in which the majority of the heat transfer occurs) in ANSYS (2005). One is the Monte Carlo (MC) and the other is the Discrete Transfer (DT) radiation model. Non-linearities in the systems due to scattering, diffuse reflection, or temperature dependency of radiation quantities are overcome by an iterative solution technique.

\section{Monte Carlo (MC) model}

The MC model treats the radiation field as a photon gas. A photon is selected from a photon source and stochastically tracked through the system until its weight falls below some minimum at which point it 'dies.' Each time the photon experiences an event, for example a surface intersection, scattering or absorption, the physical quantity of the radiation intensity is updated along the ray. Using this method, a complete 'history' of that photon in the system is generated. Many photon histories need to be generated to achieve good estimates of the physical quantity. This value can be specified by the user. The 
main computational overhead for CFX in generating a history is in tracking the photons across the domain.

\section{Discrete Transfer (DT) model}

The DT assumes that the spatial radiation gradients are relatively small and the radiation is emitted isotropically from the surfaces. The user defined parameter 'number of rays' in CFX determines the degree of spatial discretisation of the hemisphere above each finite surface element for radiation emission. Higher values mean a better representation of the reality and a higher accuracy, but at the same time will result in a significant increase in computational effort. The paths of rays are calculated only once, at the beginning of the simulation, and are then stored and re-used which leads to significant savings of computer resources.

Due to the high computational resources required for the calculation of the radiation field for the $\mathrm{MC}$ and DT radiation models, it is essential to find a trade-off between accuracy and computational effort. This is obtained using a coarser mesh for the radiation field than for the flow field assuming that the radiation field changes at a slower rate than any other transport variables (ANSYS 2005).

\section{MODEL DETAILS}

The quasi-2D model used for verification of radiation modelling using CFX contains a square cavity of $\mathrm{X}=1000 \mathrm{~mm}$ and $\mathrm{Y}=1000 \mathrm{~mm}$ which interacts thermally with an adjacent internal wall of $\mathrm{X}=220 \mathrm{~mm}$ thickness (see Figure 1). The high and low end z-planes form symmetry boundaries. The other surfaces are opaque to radiation.

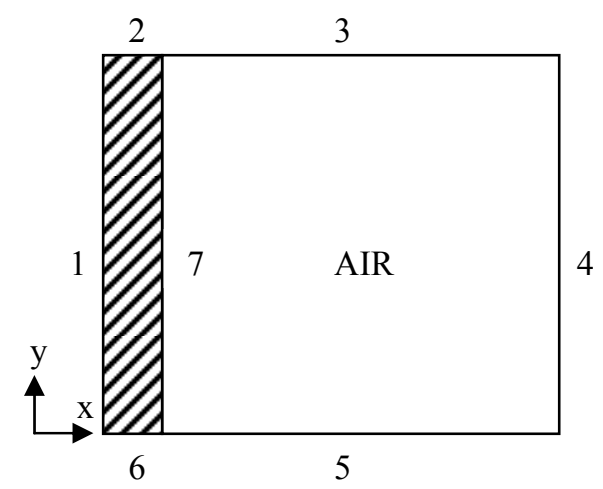

Figure 1: Room model of conjugate heat transfer. The surface numbers assign the boundary conditions specific to the cases investigated to the surfaces.

Verification has been carried out in three steps using test cases 1, 2 and 3 .

\section{Case 1}

The model in case 1 represents two parallel infinite surfaces of different temperature. The surface numbers 3 and 5 shown in Figure 1 contain symmetry conditions. The surfaces 1,2 and 6 are adiabatic and surface 4 contains an isothermal temperature of $20^{\circ} \mathrm{C}$. The thermal condition at the interface surface 7 adjusts to the conditions of the solid wall $\left(\mathrm{c}=880 \quad \mathrm{~J} /(\mathrm{kg} \cdot \mathrm{K}), \quad \rho=2300 \mathrm{~kg} / \mathrm{m}^{3}\right.$, $\lambda=1.4 \mathrm{~W} /(\mathrm{m} \cdot \mathrm{K}))$ and the adjacent air. The solid has an initial temperature of $27.5^{\circ} \mathrm{C}$ and the fluid has an initial temperature of $20^{\circ} \mathrm{C}$ throughout the domain. Radiative emission coefficients of $\varepsilon=0.9$ are considered at the enclosure surfaces. Convective heat transfer is neglected in this model (i.e. mass and momentum equations are not calculated) and only conduction and radiation heat transfer is considered.

\section{Case 2}

This model represents a room cavity enclosed by finite walls. The model boundary conditions of case 1 are modified for case 2 replacing the symmetry boundary conditions of surfaces 3 and 5 by adiabatic boundary conditions which contain a radiative emissivity of $\varepsilon=0.01$. Heat transfer due to radiation and conduction is considered as for case 1 .

\section{Case 3}

The room model for case 3 contains all aspects of heat transfer (i.e. conduction, convection and radiation). Case 2 was modified using radiation emissivity values of $\varepsilon=0.9$ for surfaces 3 and 5 and common brick material for the solid wall $\left(\mathrm{c}=835 \mathrm{~J} /(\mathrm{kg} \cdot \mathrm{K}), \quad \rho=1920 \mathrm{~kg} / \mathrm{m}^{3}, \quad \lambda=0.72 \mathrm{~W} /(\mathrm{m} \cdot \mathrm{K})\right)$. The fluid domain contains an initial temperature of $23.75^{\circ} \mathrm{C}$.

Numerical results of CFX for radiative heat exchange were verified for cases 1 and 2 using analytical solutions for two parallel plates of infinite size and for a cavity enclosed by finite surfaces, respectively. The radiative surface heat transfer for surfaces 4 and 7 was evaluated for the thermal conditions after a dynamic CFX simulation of $2 \mathrm{~h}$. Although verification could have been carried out using a steady-state solution scheme for cases 1 and 2, the transient scheme was used in order to investigate the potential application of the radiation models to future research of transient problems in terms of computational effort. To consider the conjugate heat transfer between the solid wall and the air, heat conduction was also calculated. The radiation and energy equations were calculated every time step in CFX using a time step size of $60 \mathrm{~s}$. The room model depth used in CFX for cases 1 and 2 was $Z=500 \mathrm{~mm}$. The mesh surface and core elements contained maximum edge length scales of $50 \mathrm{~mm}$ and $100 \mathrm{~mm}$, respectively. The mesh was refined close to the 
surfaces using prism inflation (first prism layer height $0.1 \mathrm{~mm}$, total number of prisms 4).

Dynamic predictions for CFX using the adaptive freeze-flow method (Zitzmann et al 2007a) were verified using the room model of case 3 . Thus, the radiation and energy equations were calculated every time step while the flow equations were frozen for variable time periods and later updated. The time step sizes for the unfrozen and frozen flow periods were $1 \mathrm{~s}$ and $60 \mathrm{~s}$ respectievely, adopting the parameter settings for the lengths of unfrozen and frozen periods from Zitzmann et al 2007a. The maximum edge length scale of the mesh in the core of the air space was reduced to a value of $50 \mathrm{~mm}$ to predict the air flow patterns more accurately in case 3. To resolve the wall boundary layer for an accurate prediction of convective surface heat transfer, the near wall region was further refined using more prism elements (16 prisms) than in case 2 . The model depth was reduced to $\mathrm{Z}=50 \mathrm{~mm}$ to reduce the additional computational effort. The dynamic temperature distribution in the room was compared with that of a traditional CFD simulation in which all equations are solved without interruption for each time step (1s). Temperatures were evaluated for different monitoring points (i.e. at $\mathrm{x} / \mathrm{X}=0.5$ at $10 \%$, $50 \%$ and $90 \%$ of the cavity height and at $\mathrm{y} / \mathrm{Y}=0.5$ in the core and the surface of the solid domain, see Zitzmann et al 2007a for a period of $12 \mathrm{~h}$. The k- $\omega$ turbulence model was applied (ANSYS 2005).

CFD simulations were considered to have converged if a residual root mean square value of $10^{-4}$ for all equations solved was achieved.

\section{RESULTS AND DISCUSSION}

The data output interval for simulations was $5 \mathrm{~min}$. All CFD simulations reached convergence as defined by the criterion above.

\section{Case 1}

Simulations were carried out using the MC model using 1,000,000 and 200,000 number of histories, and simulations were carried out using the DT model using 8 rays. The predicted maximum, minimum and average wall radiative heat fluxes after a simulation period of $2 \mathrm{~h}$ are summarised in Table 1 . For the DT model an average value of $27.7 \mathrm{~W} / \mathrm{m}^{2}$ is predicted without spatial variation. For the MC model similar average values $\left(27.8 \mathrm{~W} / \mathrm{m}^{2}\right.$ for $1,000,000$ and $27.6 \mathrm{~W} / \mathrm{m} 2$ for 200,000 histories, respectively) are predicted. However, the surface radiative heat transfer range spatially considerably for the $\mathrm{MC}$ model shown by the minima and maxima in Table 1 . This unrealistic behaviour is thought to be caused due to an insufficient long tracking of radiation particles. Figure 2 shows the distribution of radiative heat flux for the MC and the DT model which reflect the observations for Table 1 .
An average temperature of $25.8^{\circ} \mathrm{C}$ is predicted for surface 7 after two simulation hours. The analytically calculated radiative heat flux for surface 7 for these conditions is $27.7 \mathrm{~W} / \mathrm{m}^{2}$ using Eq. (2). The analytical solution compares well with the numerically predicted average values. However, the DT model is the preferable model since the MC Model showed spatially unrealistic behaviour (compare Figures 2a and $2 \mathrm{~b}$ ). Furthermore, the simulation using the DT model required less than $1 \%$ of the CPU time required by the $\mathrm{MC}$ model for the prediction of the same average values.

a)

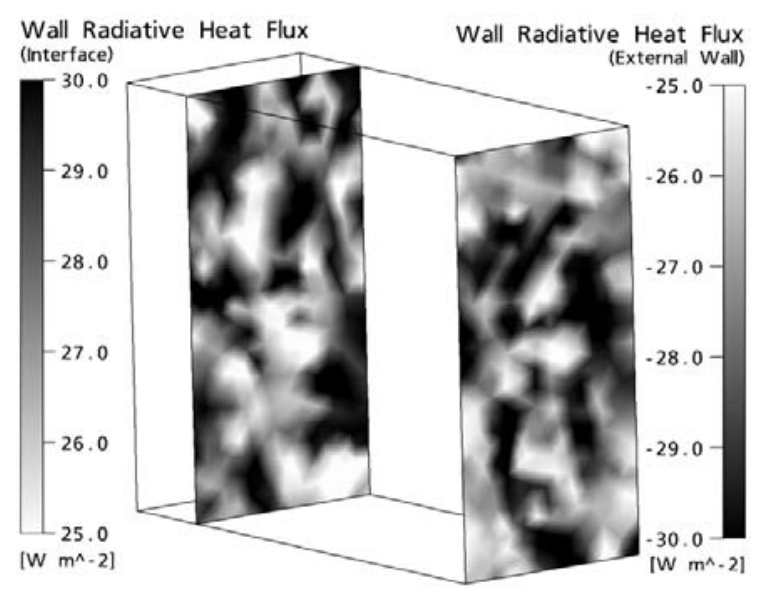

b)

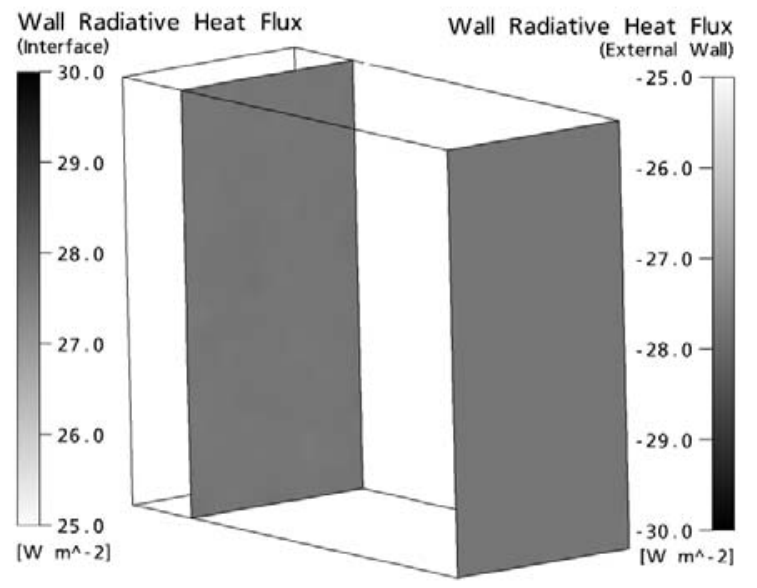

Figure 2: Case 1: Predicted wall radiative heat flux for surfaces 4 and 7 using (a) the Monte Carlo model using 1,000,000 histories and (b) the Discrete Transfer model using 8 rays.

\section{Case 2}

Predictions of the wall radiative heat flux are compared for the MC model $(2,000,000,1,000,000$ and 200,000 histories) and the DT model (8, 15, 30, 50 and 100 rays). Table 2 shows the predicted wall radiative heat fluxes after a simulation period of $2 \mathrm{~h}$. The range of values for the $\mathrm{MC}$ model using $2,000,000$ histories was about $30 \mathrm{~W} / \mathrm{m}^{2}$ and the 
average values obtained for surfaces 4 and 7 are $21.4 \mathrm{~W} / \mathrm{m}^{2}$ and $20.9 \mathrm{~W} / \mathrm{m}^{2}$, respectively. Reduced average heat transfer rates are observed for smaller numbers of histories. Similar ranges of radiative heat flux are obtained for the DT model. However, the maximum wall radiative flux is about $23 \mathrm{~W} / \mathrm{m}^{2}$ for the DT model using 30 rays and is smaller than for the MC model. Average values predicted for surfaces 4 and 7 are $21.2 \mathrm{~W} / \mathrm{m}^{2}$ and $20.7 \mathrm{~W} / \mathrm{m}^{2}$ using 30 rays. Small differences exist between the average radiative heat flux predictions of DT model simulations using less than 30 rays compared with simulations using 30 rays $\left(\Delta \dot{q} \leq 0.8 \mathrm{~W} / \mathrm{m}^{2}\right)$. The differences in average values between the emitted and absorbed radiation for the MC and DT models might be the consequence of (i) numerical inaccuracies due to mesh discretisation and equation imbalances and (ii) heat conduction considered within the transient simulation. The latter case means that the adiabatic surfaces adapt the temperatures to the adjacent air which can lead to a change of the radiative surface heat transfer at the adiabatic surfaces and therefore to a change of the radiative heat transfer at the surfaces 4 and 7 compared with the case in which no thermal conduction is included.

Figure 3 shows the distribution of the radiative heat flux at the surfaces 4 and 7. A similar distribution is mapped for the MC model as for case 1 (Figure 3a). The solution showed again an unrealistic radiative heat transfer distribution at the surfaces which is thought to be caused by an insufficiently long tracking of particles as indicated above for case 1 . However, a tendency towards smaller wall radiative heat fluxes is observed near the top and bottom where the surfaces approach the horizontal adiabatic surfaces. This behaviour is also clearly shown for the DT model (Figure 3b). Such distribution was expected since the elements close to the boundary of the surface 'see' the opposite wall with a smaller view factor than the elements in the core of the surface. Due to the higher radiative heat transfer at the core regions (see Figure 3b), the solid wall (surface 7) cools down faster than in the regions close to the horizontal adiabatic surfaces. Temperature differences of about $0.1 \mathrm{~K}$ are observed along surface 7 for a simulation using 30 rays.

The predicted average surface temperatures obtained for the MC and the DT model at surface 7 after two hours of simulation is $26.16^{\circ} \mathrm{C}$. Using Eqs. (3) and (4) a wall radiative heat flux of $22.3 \mathrm{~W} / \mathrm{m}^{2}$ is analytically calculated. For the analytical model, the surfaces are not discretised. However, the average view factors used from Wagner (1998) consider implicitly the spatial distibution of the local view factors. a)

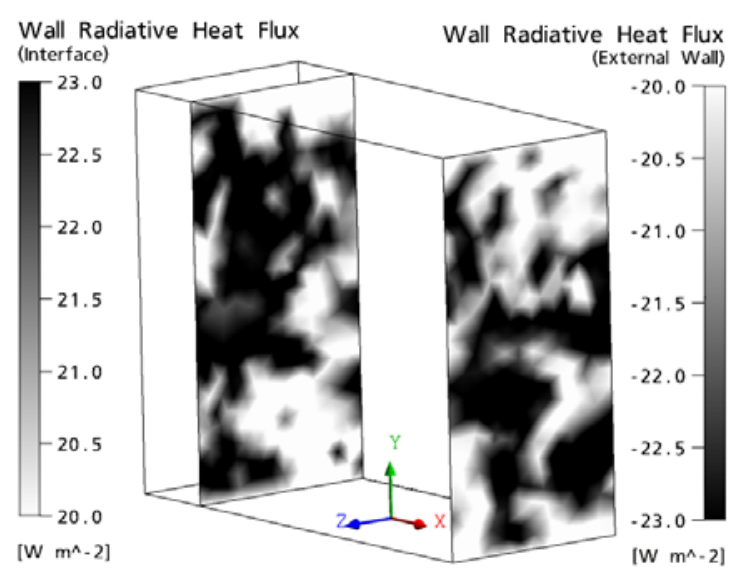

b)

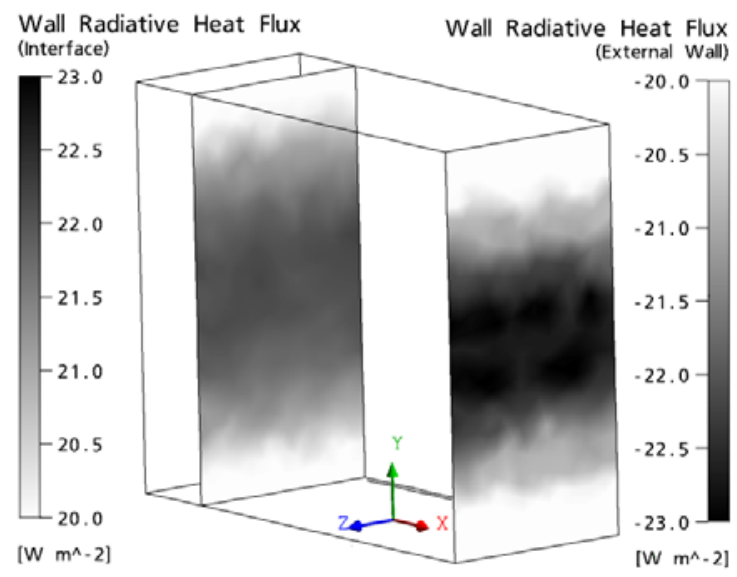

Figure 3: Predicted wall radiative heat flux for surfaces 4 and 7 using (a) the Monte Carlo model using 1,000,000 histories and (b) the Discrete Transfer model using 8 rays.

Good agreement between the MC model and the analytical data was achieved using 2,000,000 histories when comparing the average radiation heat exchange between surfaces 4 and 7 (5\% difference). Simulations using a smaller number of histories led to higher deviations. The average radiative heat transfer predictions for the Discrete Transfer model also compare favourably with the analytical solution ( $6 \%$ difference for ray numbers of 30 and more).

The small differences between the analytical solution and the numerical predictions of the MC and DT models are thought to be caused by the following:

- Inaccuracies of view factor values used for the analytical solution which were obtained from view-factor diagrams from the literature.

- The simplified use of average surface temperatures for the analytical solution. A change of the temperature difference of $0.15 \mathrm{~K}$ at the investigated temperature level for example 
leads to a change of $0.7 \mathrm{~W} / \mathrm{m}^{2}$ of the radiative wall heat exchange for the analytical calculation ( $3 \%$ deviation from the solution obtained above). This demonstrates the sensitivity of radiation heat exchange on the boundary temperatures for the analytical solution.

Taking into consideration the possible reasons considered above, the numerical predictions were considered to be satisfactory compared with the analytical solution. However, since the radiative distribution on the surfaces for the MC model is unrealistic and the simulations required high computational resources (see Table 2), this leads to the conclusion that the MC model might not be suitable for dynamic thermal building simulations. The DT model predicts the radiative distribution well and the simulation time required for the dynamic simulation is significantly reduced $(2 \%$ of CPU time for the DT model using 30 rays compared with the MC model using 2,000,000 histories). However, an increase of the number of rays leads to a significant increase of computational time for the DT model thus suggesting careful use of this parameter for timeefficient simulations.

Additional simulations using an emissivity of $\varepsilon=0.9$ instead of $\varepsilon=0.01$ for the adiabatic surfaces 3 and 5 led only to marginal changes in the numerical predictions. This corresponds with the theory that the emission coefficient at the adiabatic boundaries is irrelevant in the analytical solution. In contrast, a variation of the emmissivity at surfaces 4 and 7 led to significant changes.

\section{Case 3}

Cases 1 and 2 have demonstrated that the DT model is capable of predicting the surface heat transfer accurately and was therefore considered to be the prefered radiation model for use in this work. This model was further investigated using case 3 . Since the following inter-model comparison was conducted with the same CFD code a number of 8 rays was used instead of 30 rays to reduce the computational effort.

Figure $4 \mathrm{a}$ compares the predicted temperatures at different monitor points for a simulation period of $12 \mathrm{~h}$ using the adaptive freeze-flow method and a simulation for which the flow patterns are updated every time step (base case). The observed temperature decrease at all monitor points indicates a gradual cooling of the solid wall. The temperature predictions agree well using the adaptive freeze-flow method with those of the base case. A marginal maximum temperature difference at the end of the simulation period of less than $0.03 \mathrm{~K}$ is observed (see MP 1 at the end of a simulation period of $12 \mathrm{~h}$ ). Compared with the base case, a CPU time reduction of $84 \%$ was possible using the freeze-flow method.
Figure $4 \mathrm{~b}$ shows the temperature predictions obtained for the same room model without radiative surface heat transfer, published in Zitzmann et al (2007a). The temperatures in Figure 4a show a faster cooling than in Figure $4 \mathrm{~b}$ which indicates a noticeable influence of radiation on the overall surface heat transfer. Furthermore, the increasing temperature under-prediction observed using the adaptive freeze-flow method when radiation was not modelled $\left(\Delta \mathrm{T}_{\max }=0.15 \mathrm{~K}\right.$ at MP3 $)$ is reduced by $80 \%$ when the radiation heat transfer is included. Two reasons are thought to be responsible for the reduction in error:

- The reduction of influence of convection surface heat transfer on the thermal room behaviour due to the domination of radiative heat transfer as shown by Zitzmann et al 2007b.

- The compensation of the over-predicted cooling from convection due to a coherent decrease of radiative heat transfer (Sharma et al 2007) which subsequently occurred due to the smaller temperature difference between surfaces 4 and 7 .

a)

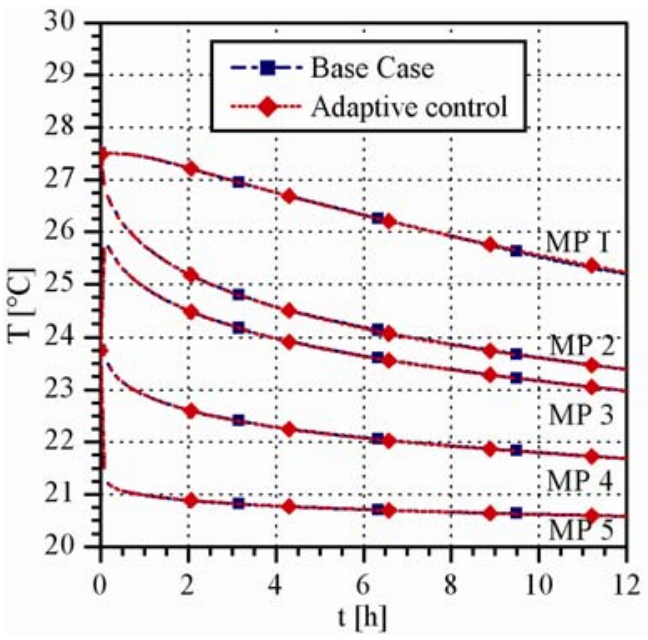

b)

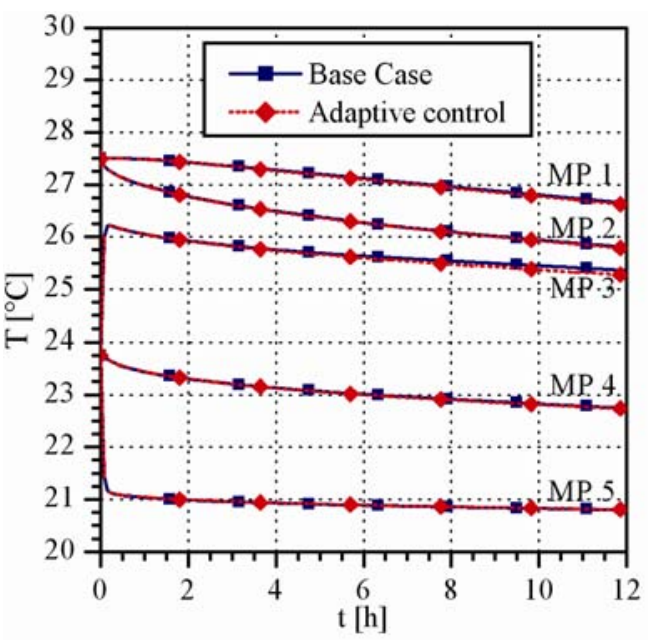

Figure 4 Temperature predictions applying the adaptive freeze-flow control method to case 3 in which radiation modelling is (a) included and (b) excluded. 
Further investigations were carried out to investigate whether the radiation equation can be 'frozen' for certain time periods to reduce the CPU time. However, for some of the room models investigated significant discrepancies were observed which led to the conclusion that the radiation equation should be calculated almost every time step.

\section{CONCLUSIONS}

Radiation modelling in spaces with high thermal mass was verified for two simple test cases using CFD. The predicted surface radiative heat transfer was compared with analytical solutions. In case 1 , the radiative heat exchange occurred between two parallel surfaces of infinite size. Case 2 contained a room enclosure with two walls of different temperature. The Monte Carlo and the DT radiation model implemented in CFX were used. The Monte Carlo model showed an unrealistic radiation distribution in all cases. The reason was thought to be the result of an insufficient number of histories used to track the reflected radiation particles through the domain. However, an increase of the number of histories led to a significant increase in computational effort. The unrealistic representation of radiative surface heat transfer and the high computational resources required led to the conclusion that the Monte Carlo model might not be suitable for dynamic thermal building simulations. The Discrete Transfer model was computational cost efficient and predictions for radiative surface heat transfer showed good agreement with analytical solutions. As a result from this study, the Discrete Transfer model is suggested as the prefered model for dynamic thermal building simulations.

In case 3 the dynamic cooling of a solid wall was investigated using the Discrete Transfer model. CFX predictions using the adaptive freeze-flow control method have been compared with a full dynamic CFX simulation in which all equations were solved at every time-step. The predicted temperatures at different monitoring points compared well with the base case. The small prediction errors which existed for investigations of Zitzmann et al (2007a) using the adaptive freeze-flow method in which radiation was neglected were reduced by $80 \%$ when radiation was considered. The CPU time was reduced by $84 \%$ using the adaptive freeze-flow control method compared with the base case.

The investigation has demonstrated that radiation heat transfer in buildings can be predicted well using the Discrete Transfer model in CFX. Furthermore, an efficient method was demonstrated for modelling dynamic thermal building simulations in which all mechanisms of heat transfer are considered using CFD by applying the adaptive freeze-flow method.

\section{ACKNOWLEDGEMENT}

This report is based on a research project which was funded by the German Ministry of Education and Research (funding NO: 1749B04). The responsibility for the content lies with the lead author of this publication.

\section{NOMENCLATURE}

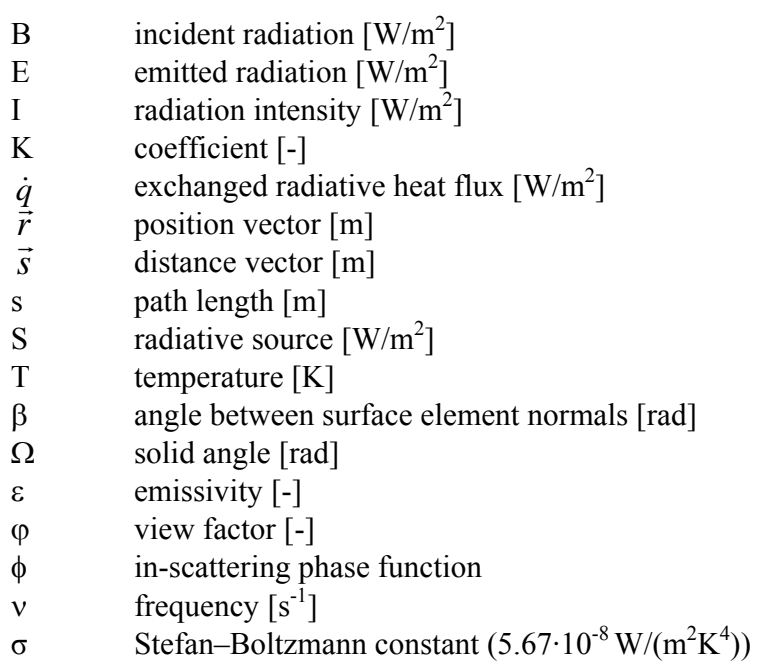

\section{INDICES}

$\begin{array}{ll}\mathrm{a} & \text { absorption } \\ \mathrm{b} & \text { black body } \\ \mathrm{i}, \mathrm{k}, \mathrm{n} & \text { surface number } \\ \mathrm{s} & \text { specular } \\ \mathrm{v} & \text { frequency }\left[\mathrm{s}^{-1}\right]\end{array}$

\section{REFERENCES}

ANSYS 2005. CFX manual. Theory. Version 10.0.

Cook M. Ji Y. and Hunt G. 2003. "CFD modelling of natural ventilation: combined wind and buoyancy forces." International Journal of Ventilation 1 (3), pp. 169-179.

Farvarolo P. Manz H. 2005. "Temperature-driven single-sided ventilation through large rectangular opening", Building and Environment 40, pp 689-699.

Ghatti V. Autif S. 2002. "Study of convective heat transfer in a radiatively cooled building using computational fluid dynamics“, Reno: Solar 2002, Nevada, 6 pages.

Groleau D. Marenne C. Raymond F. 1997. "Simulation of the night cooling effect of the night time natural ventilation: a $3 \mathrm{D}$ numerical application to the <Maison Ronde> of Botta", Athens: $18^{\text {th }}$ AIVC Conference Ventilation and Cooling (1), Greece.

Haupt W. 2001. Zur Simulation von auftriebsinduzierten Innenraumströmungen. 
Doctor thesis. Universität Gesamthochschule Kassel.

Sharma A. Velusamy K. et al. 2007. "Conjugate turbulent natural convection with surface radiation in air filled rectangular enclosures", International Journal of Heat and Mass Transfer 50, pp 625-639.

Somarathne S. Seymour M. Kolokotroni M. 2002. "Transient solution methods for dynamic thermal modelling within CFD“, International Journal of Ventilation 1 (2), pp. 141-156.

Versteeg H. Malalasekera W. 1995. Computational Fluid Dynamics. The Finite Volume Method. Essex: Pearson Prentice Hall. UK.
Wagner W. 1998. Wärmeuebertragung. Würzburg: Vogel. Germany.

Zitzmann T. Pfrommer P. and Cook M. 2007a. "Dynamisch thermsiches CFD-Verfahren mit angepasster Regelungsmethode“, Bauphysik 29 (1), pp 12-16.

Zitzmann T. Pfrommer P. and Cook M. $2007 \mathrm{~b}$. "Thermal Mass and Night-Time Ventilation using Dynamic CFD", Helsinki: Roomvent 2007, submitted.

Table 1: Predicted radiative surface heat transfer in $\mathrm{W} / \mathrm{m}^{2}$ for surfaces 4 and 7 using CFX (MC=Monte Carlo, DT=Discrete Transfer) (Case 1).

\begin{tabular}{|c|c|c|c|c|c|c|c|c|}
\hline \multirow{2}{*}{$\begin{array}{l}\text { RADIATION } \\
\text { MODEL }\end{array}$} & \multirow{2}{*}{$\begin{array}{c}\text { NUMBER OF } \\
\text { HISTORIES/RAYS }\end{array}$} & \multicolumn{3}{|c|}{$\dot{q}$ SURFACE 4} & \multicolumn{3}{|c|}{$\dot{q}$ SURFACE 7} & \multirow{2}{*}{$\begin{array}{l}\text { CPU } \\
\text { TIME }\end{array}$} \\
\hline & & MIN & MAX & AVE & MIN & MAX & AVE & \\
\hline $\mathrm{MC}$ & $1,000,000$ & -40.6 & -19.9 & -27.8 & 20.3 & 38.4 & 27.8 & 33926 \\
\hline $\mathrm{MC}$ & 200,000 & -58.9 & 24.5 & -27.7 & -100.1 & 60.3 & 27.5 & 7171 \\
\hline DT & 8 & -27.7 & -27.7 & -27.7 & 27.4 & 27.8 & 27.7 & 280 \\
\hline
\end{tabular}

Table 2: Predicted radiative surface heat transfer in $\mathrm{W} / \mathrm{m}^{2}$ for surfaces 4 and 7 using CFX (MC=Monte Carlo, DT=Discrete Transfer) (Case 2).

\begin{tabular}{|l|l|l|l|l|l|l|l|l|}
\hline \multirow{2}{*}{$\begin{array}{c}\text { RADIATION } \\
\text { MODEL }\end{array}$} & \multirow{2}{*}{$\begin{array}{c}\text { NUMBER OF } \\
\text { HISTORIES/RAYS }\end{array}$} & \multicolumn{3}{c|}{$\dot{\boldsymbol{q}}$ SURFACE 4 } & \multicolumn{3}{c|}{$\dot{\boldsymbol{q}}$ SURFACE 7 } & CPU \\
\cline { 3 - 8 } & & MIN & MAX & AVE & MIN & MAX & AVE & \multicolumn{1}{c|}{ TIME } \\
\hline MC & $2,000,000$ & -33.8 & -6.0 & -21.4 & 0 & 31 & 20.9 & 65205 \\
\hline MC & $1,000,000$ & -34.8 & -2.7 & -21.0 & 0 & 35.6 & 20.5 & 32736 \\
\hline MC & 200,000 & -46.2 & 10.9 & -20.1 & -18.4 & 56.8 & 19.9 & 6996 \\
\hline DT & 8 & -23.2 & -6.3 & -20.8 & 0.1 & 22.2 & 20.4 & 251 \\
\hline DT & 15 & -24.3 & -5.4 & -20.6 & 0.0 & 23.8 & 21.5 & 415 \\
\hline DT & 30 & -22.9 & -6.4 & -21.2 & 0.1 & 22.4 & 20.7 & 1169 \\
\hline DT & 50 & -22.9 & -6.5 & -21.2 & 0.1 & 22.4 & 20.8 & 2389 \\
\hline DT & 100 & -22.9 & -6.5 & -21.2 & 0.1 & 22.4 & 20.8 & 9110 \\
\hline
\end{tabular}

